\section{Developments in High Energy Facilities}

To learn about machine developments, Europhysics News talked to John B. Adams, head of the Division that built the $28 \mathrm{GeV}$ proton synchrotron (PS), Director-General of CERN in 1960/61, Director-General of the $400 \mathrm{GeV}$ SPS project and now Executive Director-General of CERN.

- What do you consider to have been the main advances in high energy machine construction since the early fifties?

When CERN started, the main advance up to that time had been the invention of phase stability which allowed bunches of particles to be locked to the RF accelerating system in stable buckets and CERN began the design of its synchrotron on the basis of the $3 \mathrm{GeV}$ Cosmotron scaled up to $12 \mathrm{GeV}$. Only after a visit to the U.S.A. when CERN people were made aware of the strong focusing principle, was the decision taken to build a machine that made use of this new and so far unproven technique. Calculations had suggested that if a beam encountered quadrupole magnetic fields alternating in the vertical and horizontal planes, although focusing in one plane was accompanied by defocusing in the other, under the right conditions there was a net focusing effect which meant that the aperture required for a given intensity could be kept much smaller, and for a given investment in magnets a higher energy achieved.

It was then realized that one of the major snags inherent in such a system was that the many resonances that would be encountered during the acceleration cycle could result in beam blow up and total loss. Far from there being a wide stability area in which the machine could operate, the beam would be subject to the resonances associated with the integer and half integers of the revolution frequency and also a number of non-linear resonances all of which divided up the stable area.

Nevertheless the great advantage inherent in the strong focusing principle persuaded CERN to take the risk of going straight to this "racehorse" version instead of the "enlarged cart-horse" on which work had originally begun. A lot of the success of CERN stems from that decision when the precedent for courage was established, a courage that was eminently vindicated by events; CERN was able to bring into operation the first proton accelerator based on the new principle, six months ahead of the Brookhaven machine.

Since then, advances have been less spectacular but there has been continuous improvement. Of great importance has been the work carried out in many high energy physics laboratories, and particularly at CERN on beam storage, with a view to making a colliding beam machine. The principles of stacking were worked out in the electron machine CESAR and our knowledge of beam dynamics greatly increased. At the same time, we could assess the magnitude of the technological problems involved. In an accelerator, particles remain in the machine for about $1-10 \mathrm{~s}$ whereas in a storage ring one is aiming for tens of hours. Resonances that could be ignored in the accelerator assume a new significance. Experiments on CESAR formed the basis for the decision in 1965 to build the ISR, the $31+31 \mathrm{GeV}$ proton collider that is still a unique facility.

Apart from advances in beam physics there had to be major advances in techniques, notably high vacuum, as the pressure that has to be maintained in a storage ring has to be several orders of magnitude lower than in an accelerator. Stability and accuracy of the magnetic fields had to be higher than in the PS but the crucial improvement was in the ability to get down to $10^{-10}-10^{-11}$ torr in such a large volume. At the same time our beam measurement instruments had to be much more precise if we were to be able to hold the beam for long periods.

With our next machine, the SPS, there were no fundamentally new changes; it was just very big, a property reflected in the length of time needed to arrive at the decision to build it - longer than the construction time. Nevertheless, even if, in a sense, the SPS is a scaled up version of the $\mathrm{PS}$, there was an important influence from NAL in the U.S.A. where the Fermilab machine was being built in a spirit of extreme economy. The SPS magnet system is proportionately much smaller in cross section than that of the PS. Otherwise the vacuum conditions are less exacting than those in the ISR and the RF although novel in the sense that it uses a travelling wave system was based on existing technology. The real innovation in the SPS was the computer control system which was built into the machine from the beginning and consequently affected the design of every component. This was probably the biggest technological advance that was made by CERN for this machine.

Moving to the present day we come to what is perhaps the most important invention made at CERN, representing a dramatic single step as against a steady progression. This is the stochastic beam cooling technique (see panel) which allows us to consider using big proton machines to accelerate and store antiprotons. We can, as a result, use one magnet ring to accelerate particles and antiparticles together - indeed anyone with a proton synchrotron can now contemplate using it as a proton-antiproton collider. The decision was taken at CERN about a year ago to go ahead with such an addition to the SPS. An accumulator for antiprotons is currently under construction and we are working out the system for accelerating antiparticles in the PS before injecting them into the SPS for subsequent acceleration and storage. The aim is to create a collider with a centre of mass energy of $540 \mathrm{GeV}$ - the maximum energy for which the SPS magnets can be run DC. The principles of stochastic beam cooling as well as those of electron beam cooling which was invented at Novosibirsk and is an alternative method, have both been checked experimentally at CERN in a special ring called ICE.

- What Other Technological Advances are on the Horizon?

A great effort is currently going into LEP which is a continuation of the various electron-positron machines across the world without any fundamental changes in the principles of operation. LEP is however a very big machine with a large power consumption so developments in technique are centred on these factors. They include for example the idea of concrete magnets. Maximum fields are of the order of $0.1 \mathrm{~T}$ so that the use of iron can be limited to flux shaping. Laminations are spaced out, with a type of cement filling the interval on a mark-space ratio of say $1 / 3$ or $1 / 4$. As a result magnets can be built much cheaper and as a supplementary advantage they are stiffer and so mechanically better than magnets made from stacked laminations.

Most of the power goes into the RF to compensate the synchrotron radiation losses and one has to have kilometers of cavities to supply the volts/turn needed. Two approaches are being studied to minimize the very large losses in the copper of the ca- 
vities. In the LEP machine with only four bunches circulating, there is time between bunches to transfer the energy from the accelerating cavity into a low loss cavity where it can be stored until the next bunch arrives. Alternatively the cavities can be pulsed, although this is more difficult. A big effort is also being made in several European laboratories in collaboration with CERN to develop superconducting cavities. This is a long term project and it will be some years before industry will be in a position to build such superconducting cavities with the precision and reproducibility necessary.

From time to time ideas are generated for totally revolutionary techniques of acceleration; none so far seems to offer any immediate prospect of success. Known techniques are also continually under review and linear accelerators of many kilometers in length might be attractive for electron work if superconductiviting cavities can be developed. Consideration is also being given to linear colliders but the problems here are very big. Both in the U.S.A. and the USSR superconducting magnets are now a basic component of proton machines but this implies no change in the principles of acceleration.

- At the beginning of CERN it was feared that the international laboratory would drain the Universities. This was proved not to be the case and then in 1963 the European Committee for Future Accelerators (ECFA) envisaged a pyramid of international, regional and national facilities of more or less constant proportions. This no longer corresponds to the present position. How do you see the relationship between the various sectors of the high energy physics field developing ?

Coming back to the early days of CERN, there were very few national centres; these mostly appeared afterwards. It was mainly the Universities and CERN and at the outset, teams from the Universities were expected to come with their equipment to carry out their experiments and then return to make the analysis of the results. In the middle period, there came the layer of national laboratories with intermediate energy accelerators, some of which have now closed down. The notable exceptions are electron machines which provide complementary facilities to those of CERN, or heavy ion accelerators for essentially different physics. We seem to be returning to the position that existed in the fifties with, on the one hand the Universities, and on the other the inter- national laboratories in which DESY, for example, can be included in the sense that while being a national laboratory, it is used internationally. National laboratories which no longer operate an accelerator, still play an essential rôle in constructing experiments which are then installed at the international centre.

- Is there a need for regional centres to act as intermediate data processing facilities?

Data handling has always been a problem in high energy physics and CERN has tried to avoid doing too much processing on site. It is recognized that there must be a close connexion between University teaching and research and whereas it is necessary to conduct the experiments in the international laboratory, as much of the analysis as possible should be done in the Universities. The target is to limit the fraction of the total computing effort done at CERN to about one third, although this has not yet been attained. The question of data handling is really a national problem and solutions depend on the availabity of large computers to the physics departments of the Universities. Pressure is always being exerted when regional computing facilities are inadequate for CERN to increase its capacity, but this is resisted to avoid too great a concentration here.

Sattelite data links will give the experimenters the possibility of having an almost on-line link with their base which will be an encouragement to run experiments from the base provided there are local or regional computers able to process the data. This is the key element. There is also the question of economics: we have yet to learn how much satellite links would cost in practice; so far we have been running an experiment only and we may find that the cost will be judged excessive for the majority of experimenters.

- The cost of the big machines is evidently a matter of concern to a wide section of the physics community. How much for example did the SPS cost in the end?

The agreement for its construction was for a maximum budget of $1150 \mathrm{M}$ Sw Fr at 1970 prices. The full project ended in February this year and we can now say that the overall cost is below the estimate by between 5 and $10 \%$. At the same time we have built a $400 \mathrm{GeV}$ machine (rather than 300 $\mathrm{GeV}$ ) that has even attained $500 \mathrm{GeV}$ and the experimental areas are much more elaborate than had been foreseen in the beginning. This is an old tradition in CERN to do better for less money!

- How is this record achieved when it is fairly uncommon in a national environment?

The estimates made at CERN are real; the figures are not ever knowingly below what we believe to be correct. Also we shop all over Europe and are able to choose the most economic supplier - but then other organizations do the same thing so this should not be a crucial element. Perhaps what distinguishes CERN most is the attention paid throughout the course of a project to economize as much as possible, taking advantage of new ideas that emerge. Moreover, when a contract has been placed, CERN has always worked very closely with the manufacturers of its machine components and companies knowing this do not have to add large risk factors to their estimates, as they can be confident that if unexpected manufacturing problems arise, CERN will help to find an answer

- Will there be funding for big capital projects in the future?

We cannot forecast the level of science funding in the years to come but in our planning for LEP we are assuming that the CERN Member States will not be willing to increase budgets in the future. In order to provide for LEP's construction we shall have to close down existing facilities notably the ISR and possibly the 600 $\mathrm{MeV}$ synchrocyclotron.

- This means a relatively short life for the ISR; will this be a continuing trend?

It does seem that colliders have a more limited life than fixed target machines but we have so little experience it is difficult to be dogmatic. Also one can be misled by what is really the life of a fixed target machine. The PS is twenty years old this year, but the PS of today is very different from the machine that was first built. In effect only the magnet cores and coils are the same as those that were installed originally and even then a few of the coils have had to be replaced. The main function of the PS today is to act as an injector to the SPS, which implies a quite new operational mode for this machine.

Certainly experiments are becoming bigger and of longer duration and one is likely to see a slowing down in the rate of turnover which will have some effect. But within the overall budgets it is the physics that will determine the way in which the facilities evolve and the length of time during which a given machine is considered useful. 